\title{
El quehacer de un grupo de fonoaudiólogos colombianos Educación Inclusiva
}

The work of a group of Colombian speech and language therapist.: Inclusive (n) education.

(c) (i) (2)

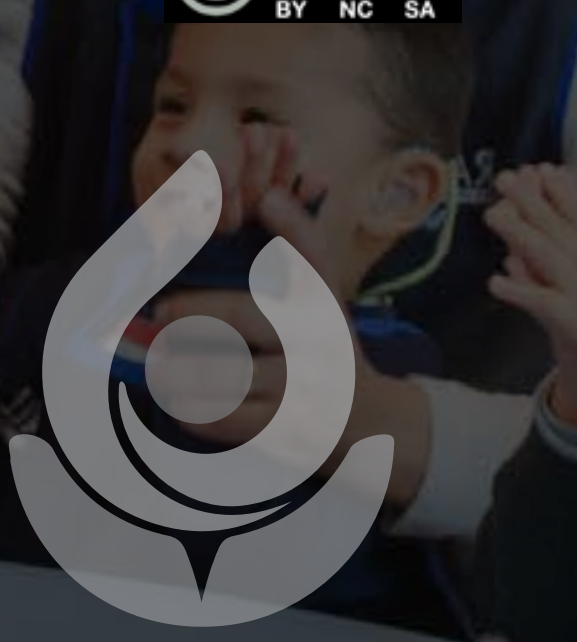

\section{Jhon Fredy Quintero Uribe}

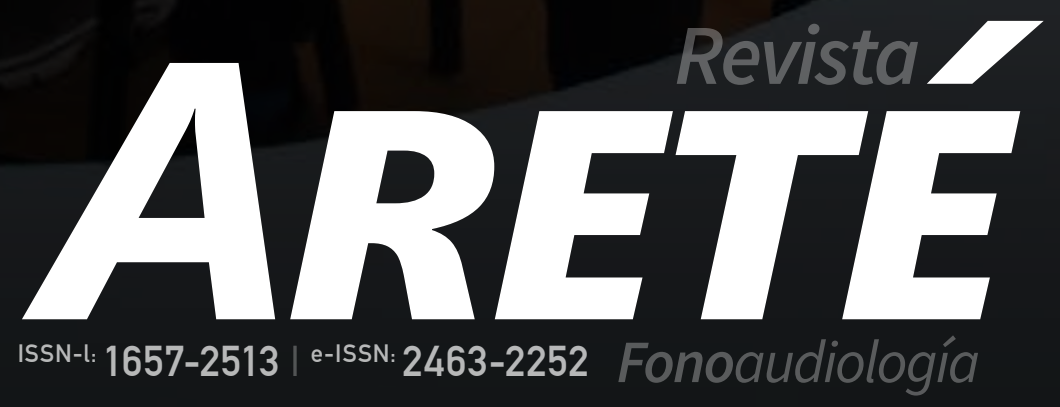




\section{ARETÉ}

ID:

1657-2513.art.19105

Title: The work of a group of Colombian speech and language therapist.

Subtitle: Inclusive education.

Título: El quehacer de un grupo de fonoaudiólogos colombianos

Subtítulo: Educación inclusiva

Alt Title / Título alternativo:

[en]: The work of a group of Colombian speech and language therapist, Inclusive education

[es]: El quehacer de un grupo de fonoaudiólogos colombianos, Educación Inclusiva.

Author (s) / Autor (es):

Quintero Uribe

Keywords / Palabras Clave:

[en]: Speech-language therapy; Speechlanguage pathology; Inclusive education; Task; Role

[es]: $\quad$ Fonoaudiología; Educación inclusiva; Quehacer; Rol

Submited: 2019-06-13

Acepted: $\quad$ 2019-06-27

\section{Resumen}

La profesión de la fonoaudiología en Colombia ha ido creciendo y con ello ha encontrado nuevos ámbitos de trabajo; uno de ellos, en educación inclusiva. Se propone describir aspectos del quehacer de un grupo de fonoaudiólogos en educación inclusiva en

Colombia en los años 2016-2017. La investigación se realizó desde un enfoque cualitativo, de nivel exploratorio y diseño de trabajo de campo a través de entrevista semiestructurada por medio virtual. Se entrevistó a 17 fonoaudiólogos de diferentes regiones del país, en su mayoría de sexo femenino, muchas de ellas con formación postgradual en áreas afines a la educación. Se encontró que los fonoaudiólogos entrevistados se encontraban vinculados la mayoría al sector educativo, aunque algunos de ellos también

a los sectores de salud y bienestar, desde los que consideran que hacen aportes a la educación inclusiva. Desarrollan su trabajo a través de un proceso de ingreso-valoración, plan de trabajoadecuaciones curriculares, intervención indirecta con asesorías e intervención directa con el escolar dentro y fuera de aula. También se encontraron actividades de docencia y administrativas. Los escenarios, los roles y responsabilidades son variados, lo que invita a seguir repensando y consolidando la profesión en educación inclusiva.

\section{Abstract}

Background and Objective: The profession of speech and language therapy in Colombia has been growing and with it has found new areas of work; one of them, in inclusive education. It is proposed to describe aspects of the work of a group of speech and language therapist in inclusive education in Colombia in the years 2016-2017. Materials and

methods: The research was conducted from a qualitative approach, exploratory level and design of field work through semi-structured interview by virtual means. Seventeen speech and language

therapist from different regions of the country were interviewed, most of them female, many of them with postgraduate training in areas related to education. Results: It was found that the speech

and language therapist interviewed are most linked to the education sector, although some of them also to the health and social sectors, from which they consider that they contribute

to inclusive education. They develop their work through a process of income-assessment, workplan curricular-adjustments, indirect intervention with advice and direct intervention with schoolchild inside and outside the classroom. There were also teaching and administrative activities. Conclusions:

The scenarios, roles and responsibilities are varied, which invites us to continue rethinking the profession in inclusive education.

\section{Citar como:}

Quintero Uribe, J. F. (2019). El quehacer de un grupo de fonoaudiólogos colombianos: Educación inclusiva. Areté, 19 (1), [pgln]-[pgOut]. Obtenido de: https://arete.ibero.edu.co/article/view/6382-2

Lic Jhon Fredy Quintero Uribe, Msc BSH

\section{Source | Filiacion:}

Fundación Universitaria María Cano

BIO:

Fonoaudiólogo, Licenciado en Ciencias Sociales, Magíster en Filosofía. Profesor-Investigador Fundación Universitaria María Cano, Colombia.

\section{City | Ciudad:}

Medellin [co]

e-mail:

jhonfredyquinterouribe@fumc.edu.co 


\section{El quehacer de un grupo de fonoaudiólogos colombianos Educación Inclusiva}

The work of a group of Colombian speech and language therapist.: Inclusive education.

Jhon Fredy Quintero Uribe

\section{Introducción}

\section{Educación inclusiva.}

La educación es un derecho expresado en la Declaración Universal de los Derechos Humanos en su artículo 26 (Organización de Naciones Unidas ONU, 2015); también en la Convención sobre los derechos del niño se obliga a las naciones a garantizar la educación a los niños en igualdad de condiciones (UNICEF, 2006)

Es el derecho a la educación lo que ayuda al ingreso de los niños y jóvenes con discapacidad a la escuela, algo que históricamente fue conocido como integración. La educación inclusiva por otra parte se plantea como una educación con apertura a todos los niños, niñas y jóvenes en etapa escolar con sus diferentes características:

En algunos países, la educación inclusiva se percibe como una modalidad destinada a atender a los escolares con discapacidad dentro del ámbito de la educación general. Sin embargo, internacionalmente se considera cada vez a nivel más amplio como una transformación que apoya y celebra la diversidad entre todos (Murguia Moré, 2016)

La educación inclusiva se define a partir de la Declaración Mundial de Educación para Todos en Jomtien-Tailandia en 1990, (UNESCO, 1990) como la universalización del acceso a la educación; esto es ratificado también en la Declaración de Salamanca de la (UNESCO, 1994), donde se expresa que la educación inclusiva implica acoger a cada escolar desde su diferencia, y que toda la comunidad educativa tiene el compromiso de participar en ello. 


\section{El quehacer de un grupo de fonoaudiólogos colombianos}

\section{Educación inclusiva}

(Booth \& Ainscow, 2000)publicaron en el año 2000 el Índex for Inclusión, donde se proponen tres dimensiones para explorar la inclusión y la exclusión en las escuelas: política, cultura y prácticas. Dicho documento se convierte en una herramienta de diagnóstico para las instituciones de educación, al ofrecer una serie de indicadores que permiten la evaluación de los avances de inclusión en las mismas; pero, por otra parte, también puede servir como guía de los aspectos que deben desarrollarse en una institución que quiera hacer inclusión. El Índex ha tenido revisiones y traducciones al español, la más reciente del año 2011 (Booth \& Ainscow, 2011)

El concepto de educación inclusiva se viene definiendo cada vez más como el derecho a la educación de todas las personas reconociendo en ellas, la diferencia y diversidad como característica esencial del ser humano y por tanto del contexto escolar mismo.

El respeto a todas las personas por encima de sus diferencias de sexo, origen, etnia, discapacidad, orientación sexual o cualquier otra circunstancia constituye un principio educativo básico. La superación de estereotipos y prejuicios sólo es posible si se posibilita el conocimiento mutuo y la interacción entre diferentes, lo cual exige que los centros recojan la diversidad social del entorno y eviten cualquier tipo de segregación escolar (Vega Fuente, 2010)

La educación inclusiva implica entonces, un reconocimiento de la diversidad y del ejercicio pleno del derecho a la educación de calidad desde las potencialidades y dificultades particulares en cada uno:

El ideal de la escuela inclusiva desde esta perspectiva -si por un momento pudiéramos detener el proceso al que antes aludíamos- sería el de un lugar en el que todos sus miembros, tanto los alumnos como los adultos, se sintieran acogidos y miembros de pleno derecho, valorados e importantes para su comunidad, donde nadie, por aprender de una forma distinta o porque tuviera características singulares de uno u otro tipo, se situara por encima o por debajo de los demás y donde estuvieran llamados a aprender lo máximo posible en relación a sus intereses, capacidades y motivaciones (Echaita \& Sandoval, 2014)

La educación inclusiva, es la educación de todos y para todos, es la educación en la diversidad, la diferencia y la colectividad. La educación inclusiva así, ya no es más la educación pensada para las personas con discapacidad.

\section{La fonoaudiología en educación inclusiva.}

La Asociación Americana de Habla, Lenguaje y Audición (ASHA American Speach-Language- Hearing, 2010), propone que el Terapeuta del lenguaje y del habla se desempeña en el sector educativo en los diferentes niveles de preescolar, básica, media y superior, identificando e interviniendo las dificultades de los escolares, apoyando las diferentes habilidades relacionadas con la lectura y la escritura, participando en equipos interdisciplinarios, contribuyendo al desarrollo del currículo y diseñando programas de prevención y promoción.

Por otra parte, (Acosta, 2006) propone una serie de responsabilidades para el logopeda en los escenarios educativos:

Ofrece información sobre la adquisición y desarrollo del lenguaje; facilita información sobre las características del alumnado con dificultades del lenguaje; participa y dinamiza la evaluación del lenguaje; participa en el diseño y desarrollo de objetivos, actividades, estrategias y materiales; intervención directa, individualizada o en

grupo; intervención dentro del aula ordinaria, conjuntamente con el profesor; participa en la toma de decisiones sobre recursos alternativos de comunicación; evalúa el progreso de los alumnos y la efectividad de los programas; trabajo colaborativo con otros profesionales y las familias (p. 22)

Rodríguez, Torres \& Vega (2017) en un estudio comparativo sobre el proceso de inclusión de escolares en la educación básica en Chile y Colombia, identifican trabajos realizados en otros países sobre el rol del fonoaudiólogo en el sector educativo, desde la percepción de docentes en instituciones educativas, y desde la percepción del profesional mismo que trabaja en dicho sector.

Desdela percepción de los docentes sobreel rol del fonoaudiólogo, encontraron el estudio de Hartas (2004) dondedocentes de instituciones educativas de Australia manifestaron la necesidad de colaboración entre profesionales en relación con el trabajo de los terapeutas del lenguaje que apoyaban niños con dificultades de comunicación y lenguaje (Rodríguez, et. al., 2017, p. 16). En este mismo sentido, docentes y terapeutas del lenguaje que manifestaron la necesidad de tener más herramientas de trabajo colaborativo en el estudio de Glover, MacCormack, \& Smith-Tamaray (2015), y sugiriendo para ello, cambios tanto en el nivel individual como institucional (Rodríguez, et. al., 2017, p. 15). También en Bangladesh, Ahmed (2015) exploró la percepción de docentes sobre la educación inclusiva y el trabajo de los terapeutas del lenguaje, manifestando necesidades de formación (Rodríguez, et. al., 2017, p. 17).

Lüdtke y Blechschmidt (2015) también determinaron la necesidad de un trabajo colaborativo con otros profesionales, especialmente los docentes de escuela, así como también formar a los terapeutas de habla y lenguaje en el proceso de inclusión educativa de niños con dificultades del lenguaje, luego del desarrollo de un trabajo especializado.

White y Spencer (2018) describen la satisfacción de coordinadores de necesidades educativas especiales (special educational needs coordinators SENCos) de 11 instituciones educativas en Reino Unido, donde se adoptó un modelo de trabajo colaborativo con terapeutas de habla y lenguaje, que incluía trabajo con los escolares con necesidades y capacitación al personal.

Sobre la percepción del rol desde los mismos profesionales, Rodríguez, et. al., (2017) encontraron estudios como el de Campell, Selkirk, \& Gaines (2016) en Canadá donde los patólogos del habla y lenguaje que trabajan en educación expresaron la necesidad de establecer cambios en el trabajo colaborativo (p. 17). Torres, Vega, \& Del Campo (2015) en Chile mostraron cómo fonoaudiólogos consideraban tener suficientes competencias en evaluación, diagnóstico e intervención, pero también requerir mayor conocimiento en los procesos de aprendizaje del lenguaje escrito en los niños para la prevención de dificultades en el mismo (Rodríguez, et. al., 2017, p. 18).

Rodríguez, et. al (2017) también describen en su trabajo, un estudio sobre las actividades propias del rol del terapeuta del lenguaje en el escenario educativo en Queensland, Australia, donde se identifican dos líneas fundamentales: intervención directa e indirecta con la población:

Los terapeutas del lenguaje realizan trabajo directo e indirecto. En el directo realizan evaluación diagnóstica, y enseñan el uso de los sistemas de comunicación aumentativa y alternativa. En el indirecto planean la terapia, colaboran con los docentes y otros profesionales, entrenan a otros en la implementación del programa, desarrollan recursos, entrevistan a los padres, se involucran en el Programa de 
Adecuaciones Educativas, desarrollan planes de apoyo individual, prescriben y revisan ayudas comunicativas, y redactan informes (Rodríguez, et. al., 2017, p. 34).

Reber (2012) en una revisión documental sobre el trabajo del terapeuta de habla y lenguaje en el sistema educativo angloamericano con niños con dificultades de lenguaje y comunicación, encontró que conceptualmente se habla de trabajo colaborativo directo e indirecto entre educadores y terapeutas; pero concluyó, que, al no encontrar otros modelos de trabajo, era importante no dejar de lado el trabajo enfocado en las necesidades individuales en lenguaje y comunicación de los escolares.

\section{Sobre el rol del fonoaudiólogo en educación inclusiva en el ámbito colombiano}

La fonoaudiología en Colombia es una profesión del área de la salud reglamentada en mediante la Ley 376 del 1997 del Congreso de la República. Se define como una profesión autónoma que trabaja por el bienestar comunicativo de la población en diferentes ámbitos y escenarios uno de los cuales es el educativo.

La fonoaudiología también fue reconocida en el año 2009 mediante el Decreto 366 del Ministerio de Educación Nacional, como una de las profesiones que haría parte del personal no docente de apoyo pedagógico en el sistema educativo. En el Artículo 10 de dicho Decreto, se enumeran una serie de responsabilidades del profesional de apoyo, las cuales en su mayoría, corresponden a acciones que permitirían la flexibilización del currículo; aunque también de manera sucinta, expresa la posibilidad de un apoyo diferenciado a algunos escolares cuando estos lo requieran (numeral 7); eso sí, teniendo conocimiento y experiencia de la población, así como certificar formación y experiencia en modelos pedagógicos, pedagogías y didácticas flexibles (Art. 14, Dec. 366- 2009).

Luego, mediante el Decreto 1075 de 2015 Único Reglamentario del Sector Educación del MEN (MEN, 2015), en su artículo 2.4.6.3.3. se plantean 3 tipos de cargos docentes, uno de los cuales tendría a cargo el apoyo a escolares con discapacidad: docentes de aula, docentes líderes de apoyo y docentes de apoyo pedagógico; este último sería el encargado de acompañar a los docentes de aula que tienen estudiantes con discapacidad.

Posteriormente, mediante el Decreto 2105 de 2017 (MEN, 2017a) en su artículo 2.4.6.3.3. sobre los tipos de cargos docentes se modifica el artículo 2.4.6.3.3. del Decreto 1075 de 2015 proponiendo como cargos: docentes de aula, docentes orientadores y docentes de apoyo pedagógico, cuyos perfiles deberían ser propuestos por el MEN mediante un manual de funciones, requisitos y competencias.

El 29 de agosto de 2017, el Ministerio de Educación Nacional de Colombia, publicó el Decreto 1421 (MEN, 2017b) mediante el cual se reglamenta la atención educativa a la población con discapacidad en los niveles de preescolar, básica y media. En esta norma se formulan nuevas herramientas para el desarrollo de la educación inclusiva tales como el PIAR (Planes Individuales de Apoyo y Ajustes Razonable). El PIAR se constituye en una herramienta del proceso, que busca proponer ajustes en el currículo a partir de la identificación del escolar con discapacidad; este documento también podría constituirse en una herramienta para la educación superior. Además, el artículo 5 de este mismo Decreto el cual modifica el artículo 2.4.6.3.3 del Decreto 1075 de 2015, se proponen nuevamente los 3 tipos de docentes que harán parte de la atención educativa: docentes de aula, líderes de apoyo y docentes de apoyo pedagógico.

Tal como queda expresado en los decretos 1075 de 2015, 2105 de 2017 y 1421 de 2017, el apoyo a los escolares con discapacidad queda a cargo del docente de apoyo pedagógico cuyo perfil sería propuesto por el MEN a través de un manual.

Sobre el perfil del rol fonoaudiológico en el sector educativo oficial, Chacón, Fajardo, Murcia y Urrego (2009) llevaron a cabo un estudio para hacer una "reconstrucción" del mismo. Para ello, exploraron el ejercicio de los profesionales de fonoaudiología que trabajaban en instituciones educativas del Distrito Capital de Bogotá. A partir del estudio, construyeron la propuesta de perfil donde se identifica el trabajo del fonoaudiólogo en sector educativo en dos escenarios esencialmente: "aula especializada" y "aula regular" (p. 94). En el perfil del fonoaudiólogo educativo en aula especializada, ubican acciones en pro del desarrollo de habilidades de comunicación en población con discapacidad, mientras, en el aula regular, ubican el trabajo con población denominada regular o sin discapacidad. Sin embargo, en el caso de la población sorda proponen que los escolares estén en aula especializada en el nivel de básica primaria, y en aula regular desde el nivel de básica secundaria. Como parte del trabajo de los fonoaudiólogos en estos escenarios, además de la intervención directa con población y procesos de asesoría a padres y profesores, también se estableció el apoyo al diseño y ajustes curriculares en las instituciones.

Álvarezy Osorno (2012) presentan los resultados de una encuestaa personal administrativo de instituciones educativas del Valle de Aburrá en Antioquia y encontraron que el $86 \%$ de ellos declaraba conocer la existencia del rol del fonoaudiólogo en sector educativo, describiendo con ello, algunas de las actividades que éste profesional realiza.

Carvajal y Cruz (2014) presentaron los resultados de la sistematización de una experiencia de atención educativa a población escolar con discapacidad en una institución de educación básica, basada en una ruta metodológica construida como parte del trabajo académico de varios años (Carvajal y Cruz, 2007). Con la sistematización proponían: "Re-construir una ruta metodológica para la identificación, diseño y adaptación de los apoyos para favorecer la participación de las poblaciones con discapacidades en la vida escolar" (p. 110). Dicha experiencia se llevó a cabo en el marco de una normativa colombiana para implementar el servicio de apoyos pedagógicos en las instituciones educativas del sector oficial (MEN, Decreto 366 de 2009), y fue desarrollada por docentes y estudiantes de programas de formación de terapia ocupacional, fisioterapia y fonoaudiología de una universidad oficial del suroccidente colombiano. Además, en el proceso, los docentes de la institución educativa objetivo tuvieron una participación muy activa.

En la sistematización se identificaron 3 etapas de desarrollo de la ruta: Identificación de un problema de participación en el desempeño escolar, evaluación colaborativa y adaptación del apoyo. Para la primera etapa, se consultaba a los docentes qué escolares habían requerido más apoyo pedagógico y luego, mediante un instrumento diseñado a partir de las categorías de actividades vitales de la CIF, estos indicaban las actividades en la vida escolar en la cual los escolares presentaban mayor dificultad. En la segunda etapa, se llevaba a cabo una evaluación colaborativa donde durante la ejecución de actividades naturales en el aula, se determinaba el desempeño real y potencial de los escolares; en esta misma etapa, el equipo de profesionales junto 


\section{El quehacer de un grupo de fonoaudiólogos colombianos}

\section{Educación inclusiva}

con los docentes, establecían el tipo de apoyos que requería el escolar. Ya en la última etapa, se implementaban los apoyos establecidos en la etapa anterior, con análisis y ajustes correspondientes.

Las autoras concluyeron que la ruta metodológica propuesta podría constituirse en una herramienta valiosa para orientar el trabajo colaborativo entre profesionales de rehabilitación y profesores, en la implementación del servicio de apoyos pedagógicos, esto es, el proceso de educación inclusiva en educación básica.

Sobre la percepción de un grupo de fonoaudiólogos colombianos acerca de su rol profesional en educación inclusiva, Quintero \& Montoya (2018) muestran en los resultados de su estudio, que dichos profesionales manifestaron que a pesar de que su rol era cada vez más conocido en el sector educativo, específicamente en educación inclusiva, no se contaba con el suficiente reconocimiento y acogida, y las condiciones de trabajo para ellos en el país seguían siendo difíciles.

También se presentan estudios sobre las concepciones de los fonoaudiólogos acerca de la discapacidad, inclusión y diversidad. En Quintero \& Osorio (2018) demostró que un grupo de fonoaudiólogos entrevistados tenían ideas actualizadas sobre los conceptos de inclusión y diversidad, pero una mirada aún deficitaria sobre el concepto de discapacidad.

No deja de ser un referente en el país, el texto de Clemencia Cuervo (1998) sobre: "La profesión de fonoaudiología: Colombia en perspectiva internacional". En este, la autora hace una breve reseña histórica sobre el trabajo del fonoaudiólogo en la educación en Colombia, y explica que los inicios del trabajo de la profesión en este sector se dan en los años 60 con niños con necesidades educativas especiales en un sistema que para la época aún era segregado ( $p$. 106). También expresa la autora que el trabajo de los fonoaudiólogos estuvo muy enfocado a la población de escolares sordos, al parecer por la falta de formación de los educadores para trabajar con estos, y porque también se identificó al fonoaudiólogo como el profesional con las mejores competencias para hacerlo (Cuervo, 1998, 103).

Cuervo presenta los diferentes momentos vividos por la fonoaudiología en el sistema educativo colombiano, desde la asignación de importantes responsabilidades que otorgaban más autonomía a la profesión en el sector, hasta las incertidumbres de ser excluidos del sistema en algunos momentos.

De esta manera, se tiene un marco normativo sobre la educación inclusiva a nivel internacional y nacional, así como algunas experiencias investigativas sobre el trabajo del fonoaudiólogo en dicho ámbito, así como breves recuentos históricos como el de Clemencia Cuervo.

\section{Metodología}

investigación con propósito exploratorio desarrollado en los años 2016 y 2017, en el que se propuso indagar algunos aspectos del ejercicio profesional del fonoaudiólogo en educación inclusiva en Colombia, entre ellos: actividades, instituciones y condiciones laborales.

\section{Participantes.}

A partir de un muestreo no probabilístico intencionado (Mayan, 2001; Sandoval, 2002) y de casos homogéneos (Sandoval, 2002, p. 123), se entrevistó a 17 fonoaudiólogos de diferentes regiones de Colombia: Bogotá, Cúcuta, Centro del Valle del Cauca, Cali, Corozal, Envigado,
Girardot, Granada (Meta), Villavicencio, Manizales y Barranquilla, que se encontraban laborando en instituciones desde las cuales se estaba haciendo un aporte a los procesos de educación inclusiva.

Gráfico 1 Género de los participantes

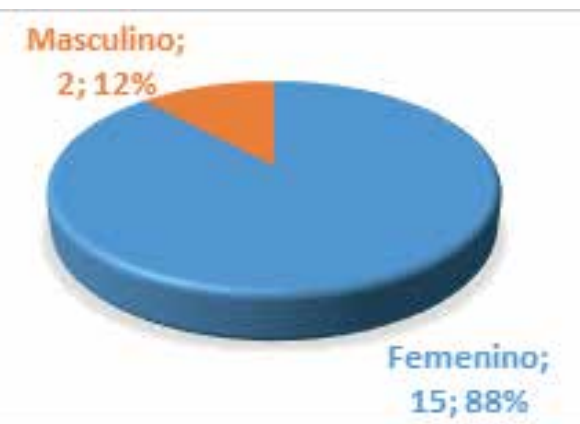

Fuente: Elaboración propia

Tal como lo muestra el gráfico 1, la gran mayoría de los profesionales de fonoaudiología que hicieron parte del estudio eran mujeres con el 88\%; mientras, tan sólo el 12\% que corresponden a 2 entrevistados, eran hombres.

Gráfico 2: Rango de edad de participantes

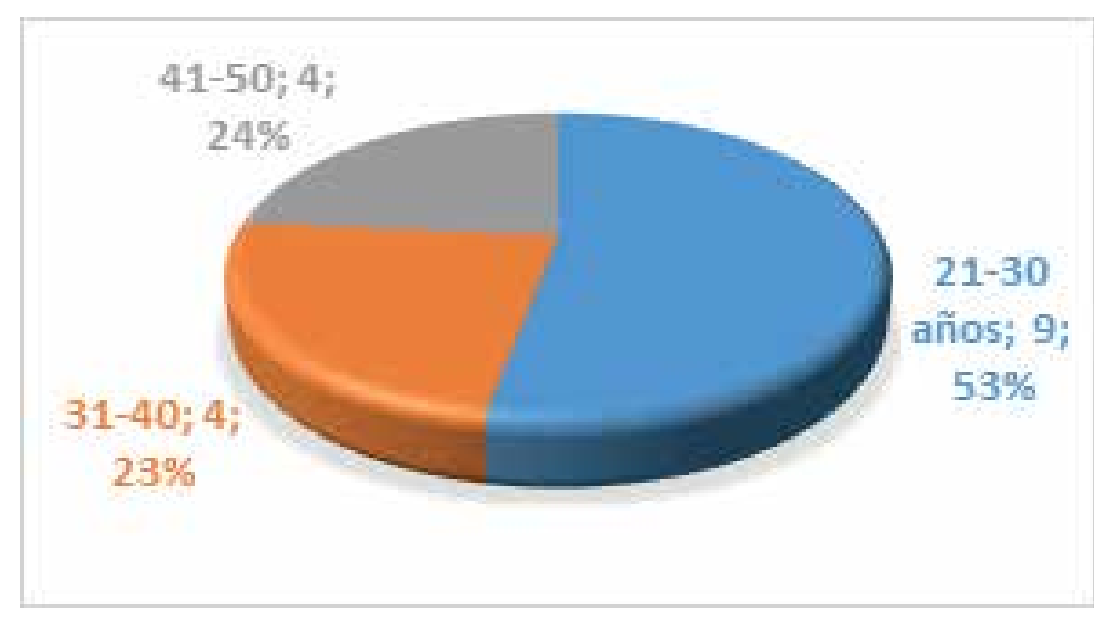

Fuente: Elaboración propia

La mayoría de los fonoaudiólogos entrevistados, 9 de los 17, estaban en el rango de edad entre 21 y 30 años; mientras, 4 estaban en el rango entre 31 y 40 años y otros 4 entre 41 y 50 años.

Gráfico 3: Años de egresado de los participantes como fonoaudiólogo

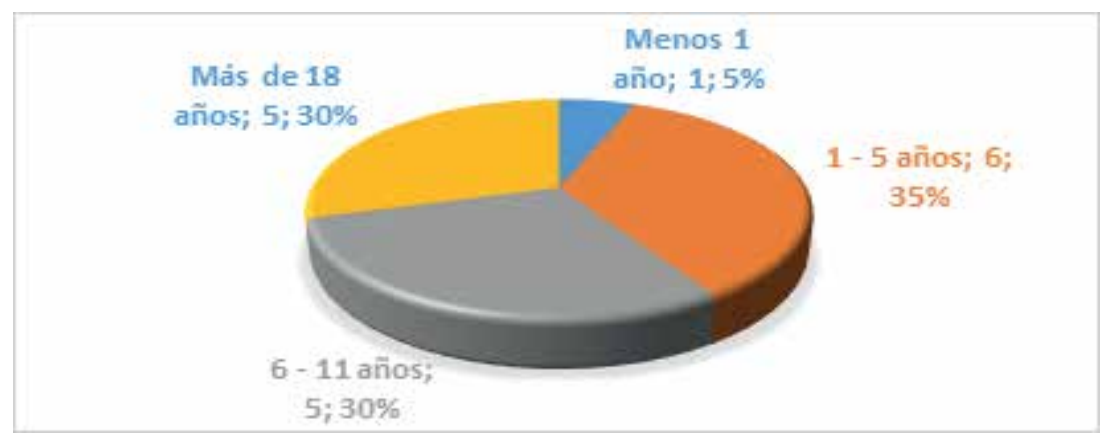

Fuente: Elaboración propia

La mayoría de los participantes, 6 del total de 17, tenía en el momento del estudio entre 1 y 5 años de egresados como fonoaudiólogos; 5 participantes tenían entre 6 y 11 años de egresados; 5 fonoaudiólogos reportaron tener más de 18 años como egresados y sólo 1 expresó tener menos de 1 año de haber obtenido su título profesional. 
Gráfico 4: Años de trabajo de los participantes en educación inclusiva

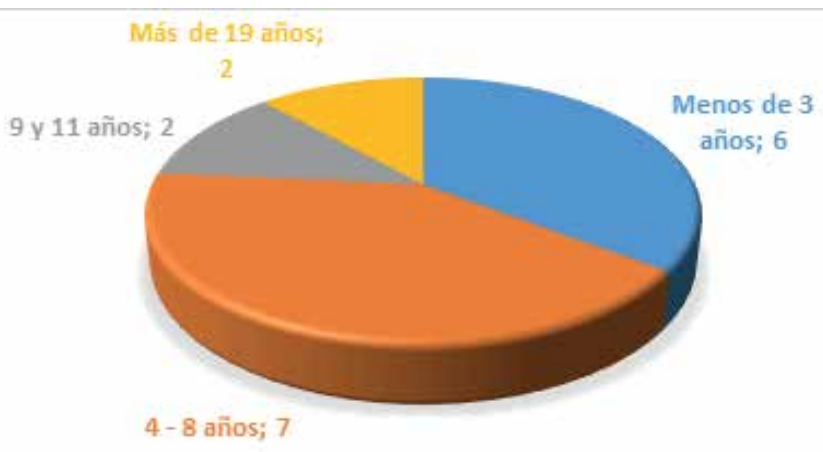

Fuente: Elaboración propia

La mayoría de los fonoaudiólogos tenía entre 4 y 8 años de trabajo en educación inclusiva, y casi en la misma proporción, 6 de ellos tenían menos de 3 años en educación inclusiva

Gráfico 5: Nivel de formación de los participantes

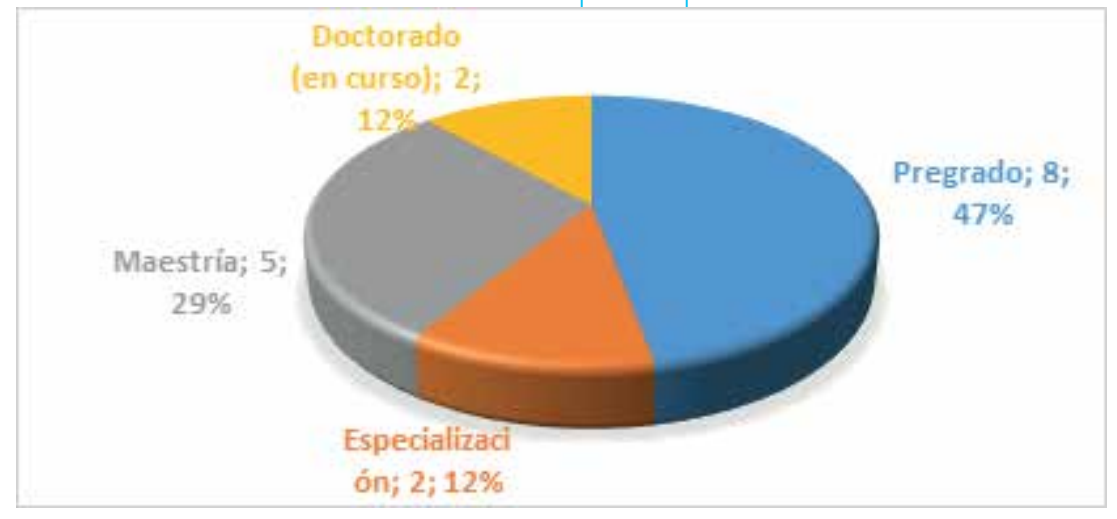

Fuente: Elaboración propia

Casi el 50\% de los entrevistados tenían nivel de formación de pregrado, y casi un tercio de ellos, nivel de formación de maestría. De los postgrados, siete eran de áreas de la educación y aprendizaje y los demás, de áreas de la salud.

\section{Instrumento.}

A partir de lo que se había explorado en estudios previos (Acosta, 2006; Chacón et. al., 2009; Rodríguez, Torres \& Vega, 2017), se diseñó una entrevista semiestructurada con 24 preguntas abiertas organizadas en 5 categorías: características institucionales, proceso de inclusión, actividades del quehacer, relación del rol con el proceso de inclusión, características laborales, y 6 preguntas demográficas. Luego de diseñado el instrumento, éste fue sometido a revisión por pares expertos a partir de lo cual se suprimieron 2 preguntas que eran redundantes con otras. En general, se quería conocer las características de las instituciones donde laboraban los fonoaudiólogos, si eran de sector educativo, salud o bienestar, oficial o privada, cómo se llevaba a cabo el proceso de inclusión de los escolares, las actividades puntuales que realizaban desde su rol, la relación de su ejercicio profesional con el proceso y sus condiciones laborales.

\section{Procedimiento.}

Durante los primeros 3 meses del estudio se realizó una convocatoria a fonoaudiólogos egresados de los programas de fonoaudiología del país, a través de sus bases de datos. La respuesta a través de dicha convocatoria no fue tan exitosa, así que luego se usó la red social Facebook y la aplicación WhatsApp para transmitirla; también se solicitó a los primeros entrevistados que referenciaran a otros colegas.
Mientras se hacía la convocatoria a los fonoaudiólogos, se realizó el diseño de la entrevista y se sometió a la evaluación de los pares expertos.

Luego de que los fonoaudiólogos se contactaban por vía telefónica con el interés de participar en el estudio, se establecía una primera cita para dar a conocer el propósito de la investigación y se acordaba un encuentro virtual a través de Skype para realizar la entrevista. Ya en el momento de la entrevista, se leía el consentimiento informado el cual previamente había sido enviado al correo electrónico del participante y este declaraba su acuerdo con el mismo. Cada entrevista duraba en promedio 60 minutos y se le explicaba al entrevistado antes de iniciar, la dinámica de esta, el tiempo estimado de duración y los temas a abordar. Las entrevistas eran grabadas en audio y se transcribían para posteriormente ser procesadas en el software estadístico.

\section{Análisis Estadístico.}

Para la interpretación de la información, se transcribieron las entrevistas y se procesaron mediante el software estadístico ATLAS.ti 7.1.0.; de esta manera, se identificaron códigos, relación entre conceptos y patrones de respuestas para lograr identificar las características del rol del fonoaudiólogo en los procesos de educación inclusiva.

\section{Resultados}

\section{Características institucionales.}

Gráfico 6: Tipo de instituciones de trabajo de fonoaudiólogos

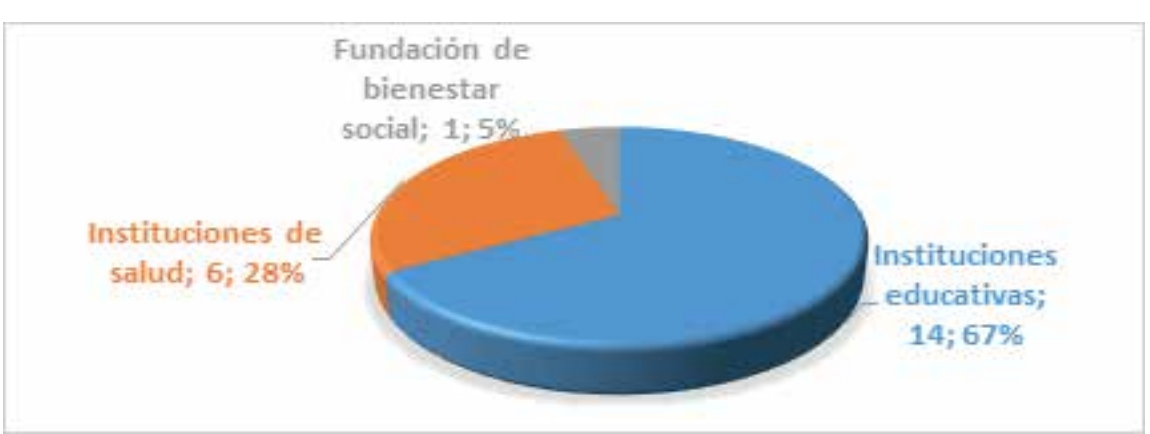

Fuente: Elaboración propia

La mayoría de los fonoaudiólogos se encontraban trabajando en instituciones educativas, de las cuales 7 eran de carácter oficial y 7 privadas; entre estas instituciones, se encontraba una institución de nivel preescolar, 11 de educación básica y secundaria y 3 universidades. Los 3 fonoaudiólogos que trabajaban como docentes en educación superior, expresaron participar en procesos de inclusión de estudiantes de pregrado.

Pero también se encontró 3 fonoaudiólogos en instituciones de salud, denominadas en Colombia como Instituciones Prestadoras de Servicios de Salud (I.P.S.), 3 fonoaudiólogos en instituciones de carácter fundación que tienen entre sus portafolios, servicios de salud contratados por la administración local, y 1 fonoaudiólogo en una institución de bienestar social de carácter fundación. Todas estas instituciones, excepto una de las de salud, eran contratadas por la administración local o por colegios privados para desarrollar programas, estrategias o el servicio de apoyo pedagógico a los escolares con discapacidad. El caso de una institución de salud se reporta porque la fonoaudióloga expresó que hacía educación inclusiva al participar en procesos terapéuticos con escolares referidos 


\section{El quehacer de un grupo de fonoaudiólogos colombianos}

Educación inclusiva

por la institución educativa.

Las características particulares en las diferentes modalidades de

De los 17 entrevistados, 2 trabajaban en 2 instituciones al mismo trabajo de los fonoaudiólogos se muestran en la tabla 1: tiempo y 1 en 3 instituciones.

Tabla 1.Características particulares en las modalidades de trabaio de los fonoaudiólogos

1 fonoaudiólogo que hacía acompañamiento en el aula a un niño que recibía tratamiento terapéutico en la metodo logía de análisis comportamental.
2 fonoaudiólogos en institución educativa oficial contratadas por la entidad territorial para el desarrollo del servicio de apoyo pedagógico.
1 fonoaudiólogo que ejecutaba el servicio de apoyo pedagógico en los colegios oficiales del municipio, desde la estrategia de aula especial, unos atendidos en las instituciones educativas y otros en la misma fundación.

1 de los fonoaudiólogos que atendía escolares remitidos 3 fonoaudiólogas docentes en educación superior por una institución educativa, con lo cual consideraba en programas de pregrado de fonoaudiología y que participaba en educación inclusiva. educación.

1 fonoaudióloga trabajando como docente en educación básica.

1 fonoaudióloga que ejerció su rol en institución privada de educación básica como coordinadora académica.
1 fonoaudiólogo que atendía niños con discapacidad en institución de educación oficial del municipio.

1 fonoaudiólogo cuya fundación tenía la modalidad de educación formal para niños con discapacidad.

1 fonoaudiólogo que trabajaba en una fundación de bienestar social que ejecutaba el servicio de apoyo pedagógico en los colegios oficiales del municipio desde el rol de coordinador.
Al preguntarles por el tipo de poblaciones que eran atendidos como parte de la educación inclusiva, se referenciaron las siguientes:

$\rightarrow$ Población sorda

$\rightarrow$ Usuarios de implante coclear o audífonos

$\rightarrow$ Baja visión

$\rightarrow$ Discapacidad intelectual

$\rightarrow$ Retraso psicomotor

$\rightarrow$ Autismo

$\rightarrow$ Asperger

$\rightarrow$ Síndrome de Down

$\rightarrow$ Otros síndromes

$\rightarrow$ Insuficiencia Motriz de Origen Cerebral IMOC (parálisis cerebral)

Los fonoaudiólogos también refirieron escolares con otro tipo de características como parte del proceso de atención: dificultades de aprendizaje, talentos excepcionales, dificultad específica de aprendizaje como dislexia y discalculia, escolares pertenecientes a poblaciones vulnerables como: comunidades negras, indígenas, víctimas de violencia sexual, víctimas del conflicto armado. En el ámbito de la educación superior, también fueron referenciados como beneficiarios de la educación inclusiva, población LGTB e inmigrantes venezolanos.

\section{Proceso de inclusión}

A partir de lo referido por los entrevistados, se reconstruyó la ruta del proceso de inclusión común en todas las instituciones

\section{Gráfico 7: Ruta proceso de inclusión}

dentificación de escolares con dificultades por parte del docente, con o sin instrumento de evaluación

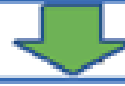

Remisión de los escolares identificados por los docentes a los profesionales de apoyo

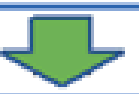

Evaluación de los escolares en aula o por fuera de ella, por parte del profesional de apoyo o equipo interdisciplinar

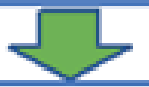

Análisis disciplinar o interdisciplinar de los resultados de evaluación

Elaboración y entrega de perfil de apoyo, plan de ajustes razonables o propuesta de flexibilización curricular a la institución y equipo docente

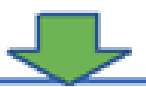

Remisión a otros profesionales o servicios en los casos en los que se determina la necesidad

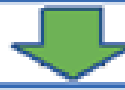

Acompañamiento o seguimiento al plan de ajustes para el escolar

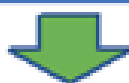

Charlas y talleres con docentes, profesionales y familia sobre estrategias de acompañamiento al proceso de inclusión

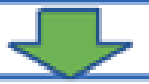

Seguimiento a la situación de aprendizaje del escolar para establecer ajustes permanentes al plan inicial 
Se encontró que, entre las actividades de los fonoaudiólogos, algunos de ellos tenían la responsabilidad de ingresar los datos de los estudiantes con discapacidad en una plataforma nacional perteneciente al Ministerio de Educación Nacional, para el registro de estudiantes en el sistema educativo colombiano, denominado SIMAT:

"[...] mi rol primero es empezar a revisar desde el SIMAT los estudiantes reportados con discapacidad. Para montarlos al SIMAT (Sistema de Matrícula Estudiantil de Educación Básica y Media SIMAT) deben tener un diagnóstico claro [...]" (P17)․․

En el caso de dos instituciones, una de salud y una fundación con servicios de salud, el niño de la institución educativa tenía que participar primero en programas de "adiestramiento" en habilidades escolares para facilitar su proceso escolar posterior. En la institución de salud, además, se intentaba que el niño primero hiciera dicho "adiestramiento" en sus instalaciones, donde se simulaban las actividades y el ambiente propio de la escuela, y cuando ya se consideraba que estaba listo para el entorno escolar, era llevado al colegio; aunque en algunos casos este proceso se hacía paralelo a la asistencia del niño en la institución educativa. En el caso de la fundación, se hacía una especie de clasificación para determinar qué niños se quedaban en sus instalaciones con apoyo terapéutico y quiénes iban al colegio:

"Entonces, si es muy habilidoso se puede pasar a un colegio... que son entre comillas regulares para ellos; si tiene más dificultades y no es tan habilidoso se trabaja desde (la fundación) [...]" (P3).

Sobre el papel de la familia en el proceso de educación inclusiva en las instituciones, seis fonoaudiólogos manifestaron que las familias estaban comprometidas con éste:

"Sí, ellos participan o están interesados... asisten, escuchan mis sugerenciasy lasacatan" (P4); "hay algunasfamilias muy comprometidas que están pendientes [...]" (P6).

De los anteriores seis fonoaudiólogos, dos manifestaron al tiempo que también había familias que no apoyaban el proceso:

"[...] hay unos que hacen el trabajo muy bien como hay papás que no tienen como mayor interés" (P5), "[...] hay otros que se van desligando del proceso" (P6).

Otros cuatro fonoaudiólogos también sentían que las familias no apoyaban muy bien el proceso:

"[...] si participan van pocos, entonces ahí es donde uno tiene que estar arreando al papa” (P7), "Muchas veces tuve que reportar casos a Bienestar Familiar. La mayoría de los casos era porque se citaba a los padres y no venían" (P14).

Para otros dos entrevistados, las familias cumplían con tareas específicas que era asignadas o hacían parte del proceso:

"[...] cuando nosotros salimos en la terapia individual vamos y le decimos: 'bueno mamá, bueno papá, mira hicimos esto durante la terapia queremos llegar a esto tu nos vas a colaborar con esto', además ellos siempre tienen un cuaderno, ese cuaderno funciona en fonoaudiología" (P3), "ellos pueden participar de algunas actividades de acompañamiento” (P9).

Por otra parte, hay tres fonoaudiólogos que expresaron el papel de la familia más desde el deber ser, es decir, desde lo que se esperaba fuera el rol de la familia en el proceso de educación inclusiva:

1 La letra/P/y el número que la acompaña, fueron los códigos asignados para identificar a cada participante, y van desde P1 a P17.
"La familia es el principal modelo" (P12), "El más importante (papel), absolutamente son la columna vertebral de cualquier proceso" (P15), "Total porque si no encontramos apoyo de la familia, el proceso no se da” (P15).

Mientras que, para dos fonoaudiólogos, se observan familias que no han hecho una aceptación o comprensión de la situación de sus hijos: "se observa mucho como la negación, no quieren aceptar la condición de su hijo y pues como no lo aceptan no lo apoyan" (P1), "la mayoría de las familias son las primeras de limitar la inclusión de sus hijos debido a que no reconocen muchas veces su cultura sorda" (P13).

\section{Actividades del quehacer}

La mayoría de las actividades reportadas por los fonoaudiólogos, consistían en el tipo de intervención indirecta (Rodríguez, et. al., 2017) a través de la asesoría a la comunidad académica y la participación en el diseño curricular y de aula.

Gráfico 8: Actividades del fonoaudiólogo en intervención indirecta en educación inclusiva:

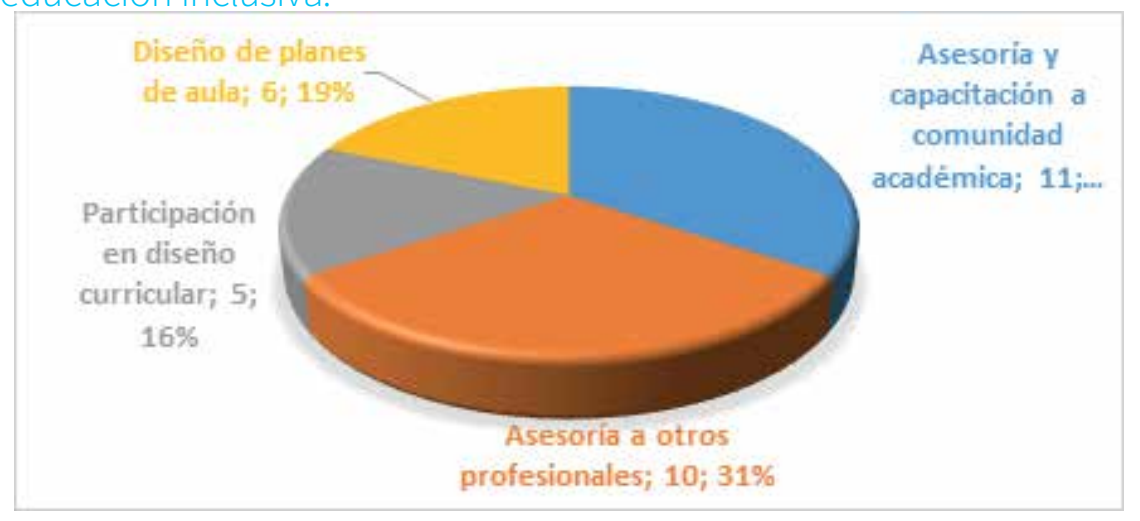

Fuente: Elaboración propia

El resto de las actividades con menor proporción, estaban dirigidas a un trabajo directo con los escolares al interior o por fuera del aula, en actividades grupales o individuales. En seis de estos casos, los fonoaudiólogos trabajaban de manera directa e individual con los niños por fuera del aula en actividades terapéutica.

En la mayoría de los casos, los fonoaudiólogos expresaron que ingresaban a las aulas para hacer valoración de los desempeños comunicativos y de aprendizaje de los niños a través de la observación no participante, para posteriormente hacer recomendaciones de apoyo a los docentes.

Como materiales de trabajo se encontró que todos usaban formatos de diseño propio, para llevar a cabo todo el proceso desde entrevista, evaluación, plan de trabajo, plan casero, formato de seguimiento, remisiones, perfiles de apoyo, formato de adecuaciones curriculares. Algunos también usaban test estandarizados para evaluación de procesos de comunicación. También usaban materiales propios del quehacer del rehabilitador tanto para evaluación como para intervención: materiales lúdicos, láminas con imágenes, cuentos.

\section{Relación del rol con el proceso de inclusión}

Se les preguntó a los participantes si creían que en las instituciones donde laboraban tenían claro su rol profesional como fonoaudiólogos, a lo que respondieron:

9 respondieron con un "sí" rotundo, que, en sus sitios de trabajo era claro su rol como fonoaudiólogos. 


\section{El quehacer de un grupo de fonoaudiólogos colombianos}

\section{Educación inclusiva}

3 expresaron que su rol profesional era claro, pero que fue un logro luego de realizar capacitaciones a docentes y demás profesionales sobre el quehacer del fonoaudiólogo: "[...] muchas profesoras no sabían ni siquiera el significado de la palabra fonoaudiólogo entonces hicimos un trabajo de sensibilización" (P10).

3 manifestaron que los profesionales que hacían parte del área de la salud o de la rehabilitación, sí tenían claro el rol del fonoaudiólogo, pero no así, los docentes o directivos de las instituciones educativas: "sí, mis compañeros los psicólogos sí tienen claro cuál es el papel, el rol del fonoaudiólogo, pero hablando de los directivos, coordinadores, demás docentes no ha sido muy claro" (P1).

1 expresó que el rol del fonoaudiólogo se confunde con otros.

1 manifestó además, que al momento de cumplir con el rol de profesional de apoyo pedagógico (Decreto 366 del 2009) en las instituciones educativas, éstas solicitaban que atendiera a los escolares en otros asuntos que no hacían parte de éste rol: "[...] en la escuela pública no... entonces remiten a chicos que son para casos específicos de convivencia que deberían ser manejados por un equipo psicosocial pero no es así, es un proceso de formación, un constante llamado a la escuela 'mira yo soy el profesional de apoyo, mi rol es este pero igual te ayudo en tu necesidad pero soy el profesional de apoyo estoy aquí por la inclusión" " (P15).

\section{Características Laborales.}

Gráfico 9: Modalidad de contrato para los fonoaudiólogos

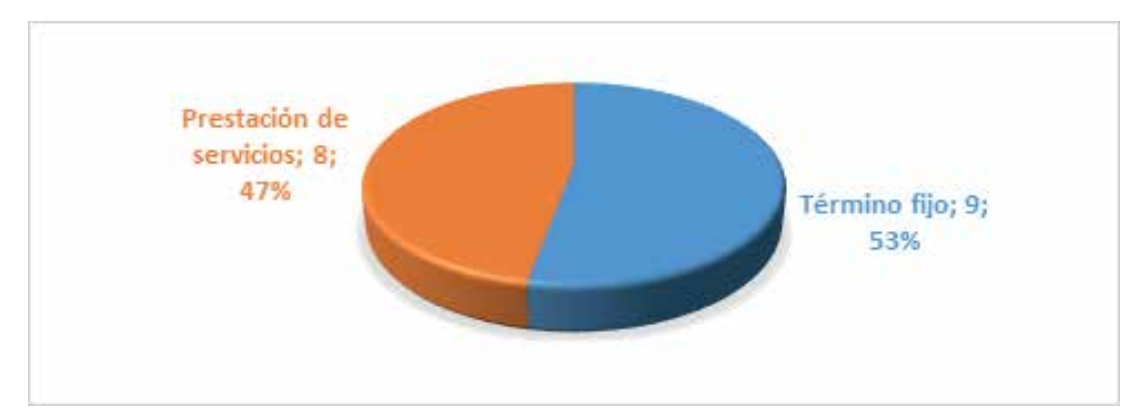

Fuente: Elaboración propia

Se encontraron diversas formas de contratación entre los fonoaudiólogos: contrato a término indefinido, contrato a término fijo y contrato por prestación de servicios de esta manera. La mayoría trabajaba con contrato a término fijo, 7 de ellos anual, 1 por 6 meses y 1 por 4 meses. De los fonoaudiólogos con contrato por prestación de servicios, 1 no tenía número de horas estipulado, 1 trabajaba 5 horas por semana, 1 trabajaba 24 horas al mes, 1 trabajaba por 2 días a la semana, 2 lo hacían por 4 horas diarias, 1 por 6 horas diarias y 1 por tiempo completo.

Gráfico 10: Remuneración económica de los fonoaudiólogos

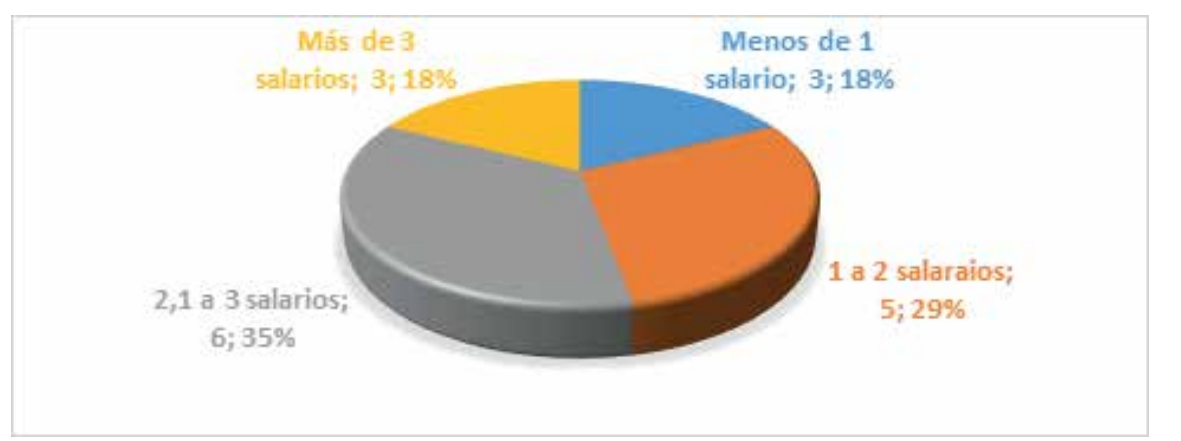

Fuente: Elaboración propia

Sobre la remuneración económica, se encontró que la mayoría ganaban entre 2,1 y 3 salarios mínimos mensuales del año 2016 en Colombia². Los 3 fonoaudiólogos que registraron salario mayor de 3 salarios mínimos mensuales correspondían a aquellos que se encontraban desempeñándose como docentes universitarios, en programas de fonoaudiología y de educación.

\section{Discusión}

Es interesante haber encontrado la diversidad de formas de trabajo que presentan los fonoaudiólogos en educación inclusiva en Colombia; ya fuera en una institución educativa o, desde instituciones que prestan servicios de salud, se consideraba que se estaba haciendo un aporte a la educación inclusiva:

"[... entonces para mí, desde mi perspectiva, la inclusión escolar no se da solamente ahí en el colegio, sino también por fuera, también desde otros contextos" (P12).

Ello lleva a la necesidad de reflexionar quizá, sobre lo que es educación inclusiva, como un paradigma de educación (MEN, 2013) que no necesariamente se circunscribe a escenarios físicos, sino con la actitud de las personas; así lo declara Echeita:

La educación inclusiva no tiene que ver, de entrada, con los lugares. Es, antes que nada, un valory una actitud personal de profundo respeto por las diferencias y de compromiso con la tarea de no hacer de ellas obstáculos sino oportunidades (Echeita, 2014, p. 107).

Desde este planteamiento todas las formas desde la cuales los fonoaudiólogos entrevistados consideraban que estaban haciendo educación inclusiva podrían ser válidas, puesto que se refieren a acciones, actividades y estrategias desde las cuales se estaría aportando a la inclusión de los escolares. Al respecto, Clemencia Cuervo, expone como estrategias de los servicios fonoaudiológicos en educación a nivel internacional los siguientes:

La visión de los servicios fonoaudiológicos en los ambientes educativos deberá tener en cuenta que su engranaje en un sistema tan complejo sólo puede ser asumido como un proceso dinámico en permanente ajuste a las necesidades y a los cambios del sistema mismo. Los desarrollos internacionales muestran que las opciones para el suministro de servicios incluyen propuestas como consultoría colaborativa, el modelo basado en el salón de clase, el modelo de trabajo por fuera del salón de clase, el suministro de servicios basados en la comunidad y la atención domiciliaria (Cuervo, 1998, p. 102)

Allí propone la autora una gama de formas de trabajo del fonoaudiólogo colombiano en educación, lo que se puede ajustar a las necesidades de trabajo en educación inclusiva; y, al juzgar por la diversidad de formas de trabajo que se expresaron en el estudio, para los fonoaudiólogos entrevistados, la educación inclusiva es más que un asunto de integración y apoyos diferenciados; se contempla al parecer, como un valor social que merece un compromiso por promover la educación para todos, desde cualquiera que sea el ámbito de trabajo.

Por ejemplo, sobre habría que indagar más sobre el rol que están cumpliendo los tantos fonoaudiólogos trabajando en educación superior, ya sea como docentes o como profesionales en programas de inclusión en diferentes facultades. En el estudio, por ejemplo, participaron tres fonoaudiólogos que eran docentes universitarios y que consideraban estar haciendo educación inclusiva, no sólo por el hecho de tener a estudiantes con discapacidad y de poblaciones 2 El salario mínimo mensual en Colombia en el año 2016 era de alrededor de
$\$ 737.717$ pesos colombianos, lo que equivalía a 239 dólares estadounidenses de
acuerdo con el promedio de la cotización del dólar estadounidense en ese año. 
vulnerables en sus clases, sino también porque sus instituciones desarrollaban planes o políticas de discapacidad e inclusión.

También resulta interesante que los fonoaudiólogos incluyeran a diferentes poblaciones como parte del marco de la educación inclusiva; es decir, no sólo a los niños con discapacidad, sino también, a niños pertenecientes a aquellas poblaciones consideradas vulnerables. Esto apunta a una concepción de diversidad no sólo como la de funcionalidad diversa o discapacidad, sino también como la que reconoce otro tipo de características;

Sobreeste mismo punto, en el momento de realización del estudio, una fonoaudióloga refirió la llegada a las instituciones educativas de niños procedentes de Venezuela como consecuencia de la situación política y social que se ha estado viviendo en dicho país, y que, para ella, eran también sujetos beneficiarios de una educación inclusiva. Esto pone en evidencia la diversidad de necesidades que afronta la institución escolar, en un mundo en el que cada vez se movilizan más personas buscando mejores oportunidades, al tiempo que nacen o renuevan tensiones étnicas, religiosas y geopolíticas.

Sobre el proceso de inclusión en el que participan los fonoaudiólogos, se tiene de manera general, un proceso que responde al esquema de atención que se ha formulado ya en algunos países de: ingreso-valoración, intervención directa e indirecta y seguimiento (Acosta, 2006; Rodríguez, et. al., 2017).

A pesar de que en su mayoría las acciones del fonoaudiólogo se concentran en la atención indirecta a través de la asesoría y formación a docentes, profesionales y familias sobre las dificultades de comunicación, la discapacidad y los apoyos diferenciados, siguen existiendo prácticas o estructuras que se desarrollan bajo la mirada rehabilitadora:

"Estoy en aulas donde solo hay niños con necesidades educativas especiales; dentro de ese proceso en realidad no se ve mucho la inclusión" (P4).

Otra actividad de intervención indirecta como sería la participación en las adecuaciones curriculares no es muy referida por los entrevistados, lo que puede estar indicando que el rol de los fonoaudiólogos en la educación sigue estando marginado a las acciones de atención especializada de los escolares (Cuervo, 1999) y no tanto en la participación del proceso de flexibilización y adaptación del currículo. Habría que preguntarse también, si los fonoaudiólogos tenemos el suficiente conocimiento de los aspectos pedagógicos y didácticos que implica la flexibilización de un currículo.

Sobre las características laborales, se encontraron porcentajes similares entre los fonoaudiólogos que tenía contratos laborales a término fijo y los que tenían contratos por prestación de servicios; mientras, sobre la remuneración económica, si se excluye el salario de los 3 docentes universitarios, los rangos salariales no parecen estar respondiendo a las expectativas que usualmente presentan los profesionales de la salud.

La predominancia de las mujeres en la profesión también se refleja en el estudio; por otra parte, la mayoría de los participantes en este estudio eran jóvenes entre 21 y 30 años; la mayoría de las participantes también trabajaban en educación inclusiva menos de 8 años, de los cuales 6 tenían menos de 3 años en dicho ámbito. Además, la mayoría también presentaba formación de pregrado.

\section{Conclusiones}

En el caso de algunos fonoaudiólogos participantes en el estudio, el aporte a la educación inclusiva se hace desde diferentes roles y escenarios de trabajo. Se puede llegar a pensar que se trata de un error metodológico el incluir perfiles que quizá no correspondían con lo que convencionalmente se considera como trabajo en educación inclusiva; simplemente no se podía excluir del estudio a aquellos profesionales que respondieron a la convocatoria, con la convicción de estar haciendo educación inclusiva; era importante escucharlos.

Las diversas formas de trabajo de los fonoaudiólogos en el estudio, y su idea amplia de los escolares que hacen parte de los procesos de educación inclusiva, quizá represente una construcción en el imaginario de estos profesionales sobre una educación ya no pensada en la discapacidad, ya no pensada en las poblaciones históricamente excluidas, sino en la diversidad. Cuestión que merece ser resaltada si se tiene en cuenta que el fonoaudiólogo es un profesional de la salud formado desde propuestas curriculares que difícilmente escapan a un paradigma normalizador.

También es de resaltar que, por lo expresado por los fonoaudiólogos, su trabajo no se restringe a la mera atención de dificultades de comunicación en los escolares, sino que, al parecer, se trasciende el objeto de estudio propio de la profesión para incorporarse al ámbito educativo desde un rol más amplio.

Lo propuesto en el Decreto 366 de 2009 donde claramente el fonoaudiólogo era reconocido como uno de los profesionales de apoyo pedagógico, queda supeditado a lo que proponen los decretos 1075 de 2015, 2105 de 2017 y 1421 de 2017, donde se expresa que será el docente de apoyo pedagógico el que estará a cargo de la atención de los escolares con discapacidad; siendo así, ¿se podría pensar que la nueva forma de participación del fonoaudiólogo en la educación inclusiva, por lo menos en la educación oficial o pública, sería mediante convocatoria docente y teniendo la formación en educación y pedagogía requerida para ello?; ¿cuál sería entonces la relación del fonoaudiólogo colombiano con la educación y la pedagogía?; ¿debe el fonoaudiólogo en Colombia desarrollar mayor formación en educación para continuar trabajando en educación inclusiva?; y si es así, ¿debería existir propuestas de formación postgradual específicas para los fonoaudiólogos que deseen trabajar en dicho ámbito? O, ¿las ya existentes desde las facultades de educación darían respuesta?

Este estudio se consideró exploratorio ya que abarcó sólo algunos aspectos de lo que es el trabajo del fonoaudiólogo en educación inclusiva; además, a partir de las experiencias de un pequeño grupo. Se considera entonces, que se debe seguir explorando el quehacer del fonoaudiólogo en este ámbito de trabajo, lo que ayudaría quizá, a definir mejor las competencias y el perfil del fonoaudiólogo en educación:

Pero no obstante haber construido una historia en el país, la fonoaudiología educativa colombiana parece no comprender aún con suficiente claridad la naturaleza de su trabajo en ambientes escolares. Esto se revela, entre otros hechos, en la ausencia de documentos escritos que precisen los conocimientos y las competencias que necesita un fonoaudiólogo para desempeñarse en el sistema educativo, al igual que las responsabilidades que puede asumir según los diferentes modelos de atención que permiten su participación (Cuervo, 1999, p. 106) 
Si bien el estudio no presenta una profundidad y alcances para generalizar la situación del fonoaudiólogo en educación inclusiva en Colombia o del terapeuta de habla y lenguaje, patólogo de habla y lenguaje o logopeda en el mundo, puede complementar los estudios ya hechos e invitar quizá, a continuar en la revisión, reflexión y trabajo colaborativo entre las diferentes propuestas curriculares de formación de profesionales de fonoaudiología en educación inclusiva.

\section{Referencias}

Acosta, V. (2006). nvestigación, evaluación y colaboración profesional ante las dificultades del lenguaje. El reto hacia los modelos inclusivos. Revista Chilena de Fonoaudiología, 7(1), 7-26. doi:10.5354/07194692.2006.48358

Álvarez, L. \& Osorno, M. (2012). Rol del fonoaudiólogo en instituciones educativas en ciclos preescolar y básica primaria. Revista Areté, Vol. $12,33-42$.

Arias, F. (2012). El proyecto de investigación. Introducción a la metodología científica (Ed. 6). Caracas: Editorial Episteme.

ASHA American Speach-Language- Hearing. (2010). Roles and Responsibilities of Speech-Language Pathologists in Schools. Obtenido de https://www.asha.org/policy/PI2010-00317/

Booth, T., \& Ainscow, M. (2000). Índice de inclusión: desarrollando el aprendizaje y la participación en las escuelas. Centre for Studies on Inclusive Education(UK). Obtenido de https://unesdoc.unesco.org/ ark:/48223/pf0000138159

Booth, T., \& Ainscow, M. (2011). Guía para la educación Inclusiva: Desarrollando el aprendizaje y la participación en los centros sociales (3 ed.). (Fuehem, Ed.) Madrid. Obtenido de file:///C:/Users/ estudiantes/Downloads/IndexLibroAgosto.pdf

Carvajal \& Cruz (2007). Ruta metodológica para la identificación, diseño y adaptación de apoyos para la participación en la escuela. Revista Areté, 7, 58-63

Carvajal \& Cruz (2014). Los apoyos para la participación del escolar con discapacidad. Revista Horizontes Pedagógicos, 16, 106-119

Chacón, J., Fajardo, L., Murcia, G., \& Urrego, A. (2009). Construcción de un paradigma de acción fonoaudiológica en la educación. Revista Areté, 9, 85-94.

Congreso de la República de Colombia (1997). Ley 376 de 1997 Por la cual se reglamenta la profesión de Fonoaudiología (Diario Oficial No. 43.079). Bogotá, Colombia.

Congreso de la República de Colombia (2013). Ley estatutaria N 1618 Por medio de la cual se establecen las disposiciones para garantizar el pleno ejercicio de los derechos de las personas con discapacidad. Bogotá, Colombia

Cuervo Echeverri, C. (1998). La profesión de fonoaudiología Colombia en perspectiva internacional. Bogotá (Colombia): Universidad Nacional de Colombia.

Echaita, G., \& Sandoval, M. (2014). Educación Inclusiva o educación sin exclusiones. Revista de Educación, 31-48. Obtenido de http:// benu.edu.mx/wp-content/uploads/2015/03/Educacion inclusiva o educacion sin exclusiones.pdf

Lüdtke, U., \& Blechschmidt, A. (2015). Speech-language therapy in inclusive school contexts: Professionalisation in the further development of speech-language pathology/logopaedics university programmes in germany and switzerland. [Sprachtherapie in inklusiven schulischen Kontexten: Professionalisierung in weiterentwickelten Studiengängen Sprachtherapie/Logopädie in Deutschland und der Schweiz] Sprache Stimme Gehor, 39(2), 86-92. doi:10.1055/s-0035-1550003
Mayan, M. (2001). Una introducción a los métodos cualitativos: Módulo de entrenamiento para estudiantes y profesionales. Canadá: Qual Institute Prees

Ministerio de Educación Nacional de Colombia (2017a). Decreto 1075 de 2015, Por medio del cual se expide el Decreto Único Reglamentario del Sector Educación. Bogotá.

Ministerio de Educación Nacional de Colombia (2017b). Decreto 1421 de 2017, Por el cual se modifica parcialmente el Decreto 2105 de 2017, Único Reglamentario del Sector Educación, en relación con la jornada única escolar, los tipos de cargos del sistema especial de carrera docente y su forma de provisión, los concursos docentes y la actividad laboral docente en el servicio educativo de los niveles de preescolar, básica y media. Bogotá.

Ministerio de Educación Nacional de Colombia (2017). Decreto 1421 de 2017, Por el cual se reglamenta en el marco de la educación inclusiva la atención educativa a la población con discapacidad. Bogotá.

Ministerio de Educación Nacional (2013). Lineamientos de la política en educación superior inclusiva. Bogotá: MEN Recuperado de: https:// www.mineducacion.gov.co/1759/articles-357277 recurso.pdf

Ministerio de Educación Nacional de Colombia. Decreto 366 de 2009, Por medio del cual se reglamenta la organización del servicio de apoyo pedagógico para la atención de los estudiantes con discapacidad y con capacidades o con talentos excepcionales en el marco de la educación inclusiva. Bogotá.

Murguia Moré, M. H. (2016). El rol del logopeda en la inclusión educativa de los escolares con trastornos de la comunicación oral. Avances en Supervisión Educativa(26), 1-12. doi:10.23824/ase.v0i26.574

Monje, C. (2011). Metodología de la investigación cuantitativa y cualitativa. Guía didáctica. Neiva: Universidad Surcolombiana

Organización Naciones Unidas ONU (2015). Declaración Universal de los Derechos Humanos. Recuperado en: http://www.un.org/es/ documents/udhr/UDHR_booklet_SP_web.pd

Quintero, J. \& Montoya, J. (2018). Percepciones de un grupo de fonoaudiólogos colombianos sobre su rol profesional en la educación inclusiva. Revista Colombiana De Rehabilitación, 17(1), 38 45. https://doi.org/10.30788/RevColReh.v17.n1.2018.301

Quintero, J. \& Osorio, M. (2018). Discapacidad, diversidad e inclusión: concepciones de fonoaudiólogos que trabajan en educación inclusiva. Revista Facultad Nacional de Salud Pública, 36(3), 52-59. https://dx.doi.org/10.17533/udea.rfnsp.v36n3a06

Reber, K. (2012). Education and therapy for children with special language and communication needs in inclusive settings: Perspectives of the network between special education, speech and language therapy and mainstream schools. [Sprachheilpädagogik und sprachtherapie in inklusiven settings: Perspektiven der vernetzung zwischen sprachheilpädagogik, sprachtherapie und regelschule] L.O.G.O.S.Interdisziplinair, 20(4), 264-275. doi:10.7345/prolog-1204264

Rodriguez, Y., \& Torres, A. (2017). Estudio comparativo del proceso de educación inclusiva en instituciones de educación básica chilenas y colombianas: Una perspectiva fonoaudiológica. Corporación Universitaria Iberoamericana. Obtenido de http:// repositorio.iberoamericana.edu.co/bitstream/001/493/1/Estudio\%20 comparativo\%20del\%20proceso\%20de\%20educaci\%C3\%B3n\%20 inclusiva\%20en\%20instituciones\%20de\%20educaci\% C3\%B3n\%20 b\%C3\%A1sica\%20chilenas\%20y\%20colombianas\%20una\%20 perspectiva\%20fonoaudiol\%C3\%

Sandoval, C. (2002). Módulo 4: Investigación cualitativa. Especialización en teoría, métodos y técnicas de investigación social. Bogotá: Instituto Colombiano para el Fomento de la Educación Superior ICFES.

UNESCO. (1990). Declaración Mundial sobre educación para todos. Marco de acción para satisfacer las necesidades básicas de aprendizaje en Jomtien, Ta i l a n d i a. New York. Obtenido de http://www.unesco. org/education/pdf/JOMTIE S.PDF 
UNESCO. (1994). Declaración de Salamanca y Marco de acción para las necesidades educativas especiales. Salamanca. Obtenido de http:// www.unesco.org/education/pdf/SALAMA_S.PDF

UNICEF. (2006). Convención sobre los derechos de los niños. Madrid: Unicef Comité Español. Recuperado el 2019, de https://www.un.org/es/ events/childrenday/pdf/derechos.pdf
Vega Fuente, A. (2010). La Educación Inclusiva: Un deber de Justicia. Revista Sociológica de pensamiento crítico, 4(1), 57-77. Obtenido de http://riberdis.cedd.net/bitstream/handle/11181/3523/la_educacion_ inclusiva.pdf? sequence=1

White, S., \& Spencer, S. (2018). A school-commissioned model of speech and language therapy. Child Language Teaching and Therapy, 34(2), 141-153. doi:10.1177/026565901 
$\frac{7}{7}$
$\frac{7}{\pi}$
$\frac{9}{2}$

递

悉

14 